\title{
Remarks on Tauberian Theorem for Quasicentral Banach Algebras
}

\author{
Sin-ei TAKahasi*
}

Let $A$ be a semisimple quasicentral Banach algebra with bounded approximate identity and suppose the center $Z(A)$ of $A$ is completely regular. We denote by $\operatorname{supp}_{B}(x)$ the hull-kernel closure of $\{P \in \operatorname{Prim} B: x \notin P\}$ whenever $B$ is an algebra with structure space $\operatorname{Prim} B$ and $x \in B . \quad \operatorname{supp}_{B}(x)$ is called the support of $x$. Also denote by $B_{00}$ the set of all $x \in B$ such that $\operatorname{supp}_{B}(x)$ is quasicompact, namely it satisfies the Borel-Lebesgue axiom without necessarily being Hausdorff. Let $\Phi$ be Dixmier's representation of $Z(M(A))$, the central double centralizer algebra of $A$ (see [4] for definition) and $Z_{00}(A)$ the set of all $z \in Z(A)$ such that $\operatorname{supp}\left(\Phi_{L z}\right)$ is quasicompact. Here $L z(x)=z x, x \in A$ and $\operatorname{supp}\left(\Phi_{L_{2}}\right)$ is the hull-kernel closure of $\left\{P \in \operatorname{Prim} A: \Phi_{L z}(P) \neq 0\right\}$. Actually $Z_{00}(A)=A_{00} \cap Z(A) \subset(Z(A))_{00}$ (cf. [5, Lemma 3.4 and 3.7]). However $Z_{00}(A)=(Z(A))_{00}$ was left open in [5]. The following result asserts that this is true.

Lemma. $Z_{00}(A)=(Z(A))_{00}$.

Proof. We have already shown that $Z_{00}(A) \subset(Z(A))_{00}$ as stated above. To show the converse inclusion, let $z \in(Z(A))_{00}$ and $\left\{P_{\lambda}\right\}$ a net in $\operatorname{supp}\left(\Phi_{L z}\right)$. Set $\sigma(P)=P \cap Z(A)$ for each $P \in \operatorname{Prim} A$. In the proof of [5, Lemma 3.7], it can be observed that $\sigma\left(\operatorname{supp}\left(\Phi_{L z^{\prime}}\right)\right) \subset \operatorname{supp}_{z(A)}\left(z^{\prime}\right)$ for all $z^{\prime} \in Z(A)$. Then $\left\{\sigma\left(P_{\lambda}\right)\right\}$ is a net in $\operatorname{supp}_{Z(A)}(z)$ and hence there exists a convergent subnet $\left\{\sigma\left(P_{\lambda^{\prime}}\right)\right\}$ of $\left\{\sigma\left(P_{\lambda}\right)\right\}$ because $\operatorname{supp}_{z(A)}(z)$ is compact. Let $M$ be a limit point of $\left\{\sigma\left(P_{\lambda^{\prime}}\right)\right\}$. By the complete regularity of $Z(A)$, there exists a neighbourhood $U_{M}$ of $M$ such that $\operatorname{ker}\left(U_{M}\right)$ is modular. We can assume, without loss of generality, that $\sigma\left(P_{\lambda^{\prime}}\right) \in U_{M}$ for all $\lambda^{\prime}$. We next show that $\mathrm{cl}\left(\sigma^{-1}\left(U_{M}\right)\right)$ is quasicompact ("cl" denotes the hull-kernel closure.) In fact let $P$ be an arbitrary point in $\sigma^{-1}\left(U_{M}\right)$. By the quasicentrality of $A$ and [3, Lemma 2.7.7], there exists $e_{p} \in Z(A)$ which is an identity for $A$ modulo $P$. Since $\sigma(P) \in U_{M}$, it follows that $e_{P}(1-e) \in \operatorname{ker}\left(U_{M}\right) \subset \sigma(P) \subset P$, where $e$ is an identity for $Z(A)$ modulo $\operatorname{ker}\left(U_{M}\right)$. Note also that $e\left(1-e_{P}\right) \in P$. Therefore $e-e_{P}$ must be in $P$ and hence

Received March 15, 1986.

Research partially supported by the Grant-in-Aid for Scientific

Research C-60540007 from the Ministry of Education.

* Department of Mathematics, Ibaraki University, Mito, Ibaraki 310, Japan. 


$$
A(1-e) \in A\left(1-e_{P}\right)+A\left(e_{P}-e\right) \subset P+A P=P .
$$

We then conclude that $\operatorname{ker}\left(\sigma^{-1}\left(U_{M}\right)\right)$ is modular and so $\operatorname{cl}\left(\sigma^{-1}\left(U_{M}\right)\right)$ is quasicompact. Now since $\left\{P_{\lambda^{\prime}}\right\}$ is a net in $\sigma^{-1}\left(U_{M}\right)$, it has a convergent subnet whose limit point is in $\operatorname{supp}\left(\Phi_{L_{z}}\right)$. Thus $\operatorname{supp}\left(\Phi_{L_{z}}\right)$ must be quasicompact. In other words, $z \in Z_{00}(A)$ and the proof is complete.

If $B$ is a Banach algebra and $B_{00}$ is norm dense in $B$ then $B$ is said to be Tauberian. Note that if $Z(A)$ is Tauberian then $Z_{00}(A)$ is norm dense in $Z(A)$ from the above lemma. Therefore the first half of [5, Theorem 3.5] is improved as follows:

THEOREM. If $Z(A)$ is Tauberian then every closed two-said ideal of $A$ which does not contain $Z(A)$ is contained in some primitive ideal of $A$.

Let us now regard $A$ as a Banach $Z(A)$-module and suppose that $A$ is essential (cf. $[2,15.1]$ for definition). Then $A$ is obviously quasicentral. Moreover by the above theorem, if $Z(A)$ is Tauberian then every proper closed two-sided ideal of $A$ is contained in some primitive ideal of $A$ and hence $A$ is also Tauberian because hull $A_{00}=\emptyset$ from [5, Lemma 3.4].

However we don't know whether the quasicentrality of $A$ implies the essentiality of $A$ or not. We know only that if $A$ is quasicentral then $A$ is order-free, namely $Z(A) x=\{0\} \Rightarrow x=0$ for $x \in A$ and that if $A$ is a $C^{*}$-algebra then the quasicentrality of $A$ implies the essentiality of $A$ (see [1, Proposition 1]).

\section{References}

[1] R. J. Archbold, Density theorems for a center of a $C^{*}$-algebra, J. London Math. Soc. (2) 10 (1975), 187-197.

[2] R.S. Doran and J. Wichmann, Approximate identities and factrization in Banach modules, Lecture Note in Math. \$768, Springer-Verlag Berlin Heidelberg New York (1979).

[ 3 ] C. E. Rickart, General Theory of Banach Algebras, Van Nostrand, N. J. (1960).

[4] S. Takahasi, Dixmier's representation theorem of central double centralizers on Banach algebras, Trans. Amer. Math. Soc. 253 (1979), 229-236.

[5] S. Takahasi, Central double centralizers on quasi-central Banach algebras with bounded approximate identity, Can. J. Math. 35 (1983), 373-384. 